Version Date: May 30th, 2005

Title:

\title{
Fast, Preisach-like characterization of hysteretic systems
}

\author{
Authors: \\ Marek W. Gutowski ${ }^{1}$, Lajos K. Varga ${ }^{2}$ and Attila Kákay ${ }^{2}$ \\ ${ }^{1}$ Institute of Physics, Polish Academy of Sciences, Al. Lotników 32/46, 02- \\ 668 Warszawa, Poland \\ ${ }^{2}$ Research Institute for Solid State Physics and Optics, Hungarian Academy \\ of Sciences, P.O.Box 49, H-1525 Budapest, Hungary \\ email: Marek.Gutowski@ifpan.edu.pl
}

\begin{abstract}
Proposed is a substantially simplified, Preisach-like model for characterization of hysteretic systems, in particular magnetic systems. The main idea is to replace a two-dimensional Preisach density with just two real functions, describing in a unique way the reversible and irreversible processes. As a byproduct of our model we prove, that the major hysteresis loop alone is insufficient to produce the unique Preisach map.
\end{abstract}

Keywords:

magnetic hysteresis; magnetization processes; materials testing; modeling; 


\section{Introduction}

One of the most widely known classes of hysteresis models is the Classical Preisach Model (CPM) and a rich variety of its close relatives.

The main motivation behind our work was clear separation of reversible and irreversible components of isothermal, quasi static hysteresis loops. In CPM, the reversible part is represented by hysterons located on the edge of the Preisach half-plane, i.e. on the line $H_{\uparrow}=H_{\downarrow}$ (up- and down-switching fields equal to each other). All other physically valid hysterons, i.e. those with $H_{\uparrow}>H_{\downarrow}$, are related to the irreversible magnetization processes. Usually, the Preisach distribution is interpreted in probabilistic terms. There is, however, a problem with credible characterization of the (ferro)magnetic samples with diamagnetic components, like, for example the graphite foam [1] and - of course - superconductors. Their Preisach maps may go negative in the reversible part, thus such processes should be treated separately from the rest of the complete Preisach distribution. Disregarding this distinction leads frequently to puzzling results: the negative amplitudes appear in the Preisach plane, even for ferromagnetic samples. Findings like that have been signalled since at least ten years. Until recently, they were usually attributed to experimental uncertainties or inaccuracies introduced during the data processing. Yet, even the FORC diagrams introduced by Pike [2], which are more or less equivalent to CPM, exhibit such a feature quite often. Moreover, the very existence of composite materials with negative remanence [3] constitute the unquestionable experimental evidence, that the probabilistic interpretation of the Preisach distribution is untenable. 


\section{Model description}

Inspecting results, similar to those presented by Pike and by other authors [4, we decided to try the following idea. We drastically reduce the support of the Preisach distribution, $\varrho\left(H_{\uparrow}, H_{\downarrow}\right)$, leaving for it only two lines: $H_{\uparrow}=H_{\downarrow}$ for reversible part, and $H_{\uparrow}=-H_{\downarrow}$, the mirror symmetry axis of the usual Preisach distribution, for irreversible part. This way we can separate both kinds of magnetization processes. Formally:

$$
\begin{aligned}
\varrho\left(H_{\uparrow}, H_{\downarrow}\right) & =\varrho_{\mathrm{rev}}(H) \cdot \delta\left(H_{\uparrow}-H_{\downarrow}\right) \\
& +\varrho_{\text {irr }}(H) \cdot \delta\left(H_{\uparrow}+H_{\downarrow}\right),
\end{aligned}
$$

where $\varrho_{\text {rev }}(\cdot)$ and $\varrho_{i r r}(\cdot)$ are real, single-valued functions of a single variable, and $\delta(\cdot)$ is Dirac's delta.

In this respect our idea is similar to the one presented earlier in [5]. The separate treatment for the reversible processes, using different arguments than ours, was pointed out in many papers, maybe most convincingly in [6].

\subsection{Extracting $\varrho_{\mathrm{rev}}(\cdot)$ and $\varrho_{\mathrm{irr}}(\cdot)$ from experimental data}

Our calculations strictly follow the usual path of all the Preisach-like models.

For $H<0$, the function $\varrho_{\mathrm{rev}}(H)$ is immediately identified as $\varrho_{\mathrm{rev}}(H)=$ $d M(H) / d H$, with the derivative being taken along the ascending branch of the major hysteresis loop, while for $H>0$ we have to use the identity $\varrho_{\mathrm{rev}}(H)=\varrho_{\mathrm{rev}}(-H)$.

The other function is again given by the derivative:

$\varrho_{\text {irr }}(H \geq 0)=-d / d H\left[M_{d}(H)-M_{a}(H)\right]$. Here $M_{d}$ describes the descending branch of the major hysteresis loop, and $M_{a}$ - its ascending branch. 


\subsection{Repairing the unphysical results}

The experience shows, that sometimes $\varrho_{\text {irr }}(H)$ happens to be negative on some interval, see Fig. 4 near $H=250$ Oe. This is, as a rule, accompanied by the presence of the similarly shaped, but positive, part of the function $\varrho_{\text {irr }}(\cdot)$ located at higher fields. To get rid of the 'unphysical' parts of $\varrho_{\text {irr }}(\cdot)$, we can apply the relation:

$$
\begin{array}{r}
H y(a,-a)-H y(b,-b)= \\
H y(b,-a)+H y(a,-b)-H y(b, b)-H y(-b,-b)
\end{array}
$$

where $H y(a, b)$ represents an ordinary Preisach hysteron with $H_{\uparrow}=a$ and $H_{\downarrow}=b$. From the above identity we can conclude, that essentially it might be possible to 'repair' the unphysical results, at the cost of restoration of the full Preisach model. Unfortunately, this can be done in infinitely many ways. Accidentally, we have just shown, that the Preisach map obtained from major hysteresis loop alone is not unique.

\section{Advantages and drawbacks of the model}

First of all, our model is unique - by construction. Secondly, it compares very favorably to others, when it comes to computational complexity, accuracy and experimental effort needed for data acquisition. We only use first derivatives of the original data, what introduces definitely less noise than other techniques relying on higher derivatives. The calculations are straightforward and do not include any iterative schemes. Given perfect data, the procedure reproduces them exactly. There is no need to guess, or to assume, any particular shape of the anhysteretic part of the magnetization process first, as it is required in Jiles-Atherton model [7]. 
The clearly visible drawback of our method is that the (over?)-simplified Preisach distribution cannot be interpreted in usual terms, namely as a probability distribution. As of today, we cannot offer its precise alternative interpretation, in well defined, physical terms. The ability of our model to reproduce minor loops has not been tested yet.

\section{Discussion}

The simplified procedure, as being rather simple, fast and unique, may be regarded as a new, valuable tool of analysis of magnetic (and maybe superconducting as well) systems, including non-homogeneous ones, like those containing nanocrystals, multilayer structures, patterned media, etc. The results produced with this approach should probably be interpreted in the same spirit, as those obtainable from FORC diagrams, that is, in the language of the statistics of the effective internal fields, including their signs, not just amplitudes.

Especially interesting is the shape of $\varrho_{\text {rev }}(H)$, sometimes exhibiting more than one-peak structure, thus clearly deviating from the usually assumed Langevin's formula. We suspect its close relationships with internal field distributions, investigated numerically in [8], for systems with long-range, dipolar interactions.

\section{References}

[1] H. Pardo et al., cond-mat/0407303.

[2] Ch.R. Pike et al., J. Appl. Phys. 85 (1999) 6660.

[3] Soon Cheon Byeon et al., IEEE Trans. Magn. 40 (2004) 2359. 
[4] P. Postolache et al., IEEE Trans. Magn. 39 (2003) 2531.

[5] Sergey Langwagen, cond-mat/0103573

[6] O.V. Perevertov, J. Phys. D: Appl. Phys. 35 (2002) 2467.

[7] D.C. Jiles and D.L. Atherton, J. Magn. Magn. Mater. 61 (1986) 48.

[8] Francisco J. Morentin et al., J. Appl. Phys. 93 (2003) 6641. 
List of figure captions:

Fig. 1:

Room temperature major hysteresis loops of $\mathrm{FeZrB}_{12} \mathrm{Cu}$ nanocrystalline material annealed at: $100^{\circ} \mathrm{C}, 180^{\circ} \mathrm{C}, 240^{\circ} \mathrm{C}, 360^{\circ} \mathrm{C}$ and $440^{\circ} \mathrm{C}$. Coercivity increases with increasing annealing temperature.

Fig. 2:

Decomposition of the major hysteresis loop (dotted line) into reversible and irreversible components (solid lines). Sample annealed at $100^{\circ} \mathrm{C}$.

Fig. 3:

Reversible parts, $\varrho_{\text {rev }}(H)$, of hysteresis loops shown in Fig. 1 diminish with increasing annealing temperature. Note the composite, non-Langevin shape after low-temperature annealing.

Fig. 4:

Irreversible parts of hysteresis loops, $\varrho_{\text {irr }}(H)$, shown in Fig. 1. The increasing annealing temperature activates the hysterons with higher coercivity. Note the regions with negative amplitudes followed by "regular" ones. Amplitudes expressed in the same, arbitrary units, as in Fig. 3. 


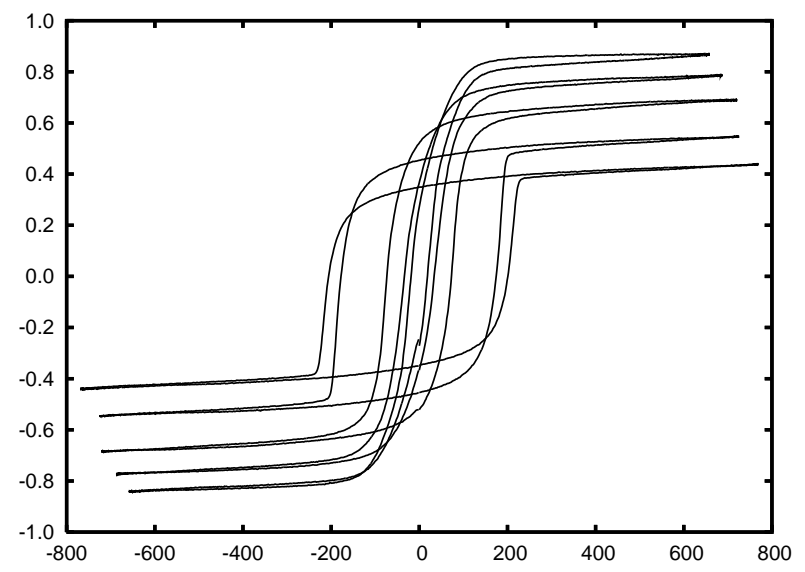

Figure 1: Room temperature major hysteresis loops of $\mathrm{FeZrB}_{12} \mathrm{Cu}$ nanocrystalline material annealed at: $100^{\circ} \mathrm{C}, 180^{\circ} \mathrm{C}, 240^{\circ} \mathrm{C}, 360^{\circ} \mathrm{C}$ and $440^{\circ} \mathrm{C}$. Coercivity increases with increasing annealing temperature.

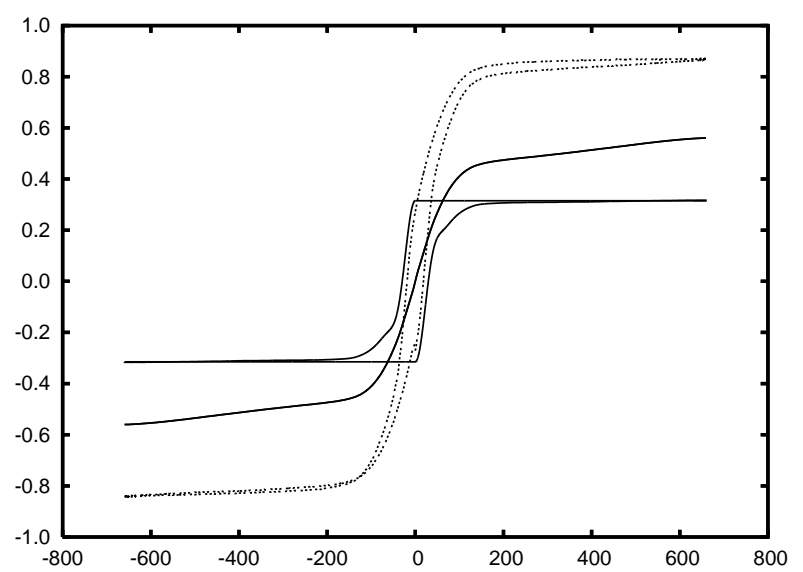

Figure 2: Decomposition of the major hysteresis loop (dotted line) into reversible and irreversible components (solid lines). Sample annealed at $100^{\circ} \mathrm{C}$ 


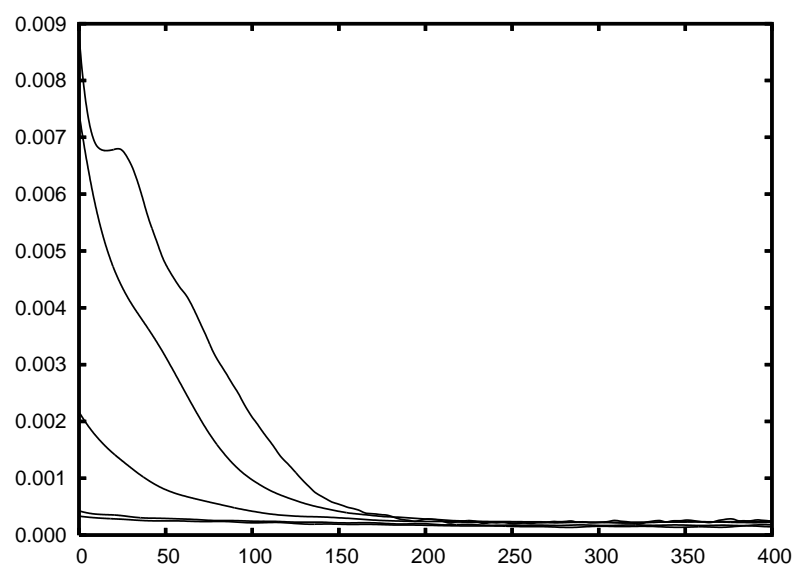

Figure 3: Reversible parts, $\varrho_{\text {rev }}(H)$, of hysteresis loops shown in Fig. 1 diminish with increasing annealing temperature. Note the composite, nonLangevin shape after low-temperature annealing.

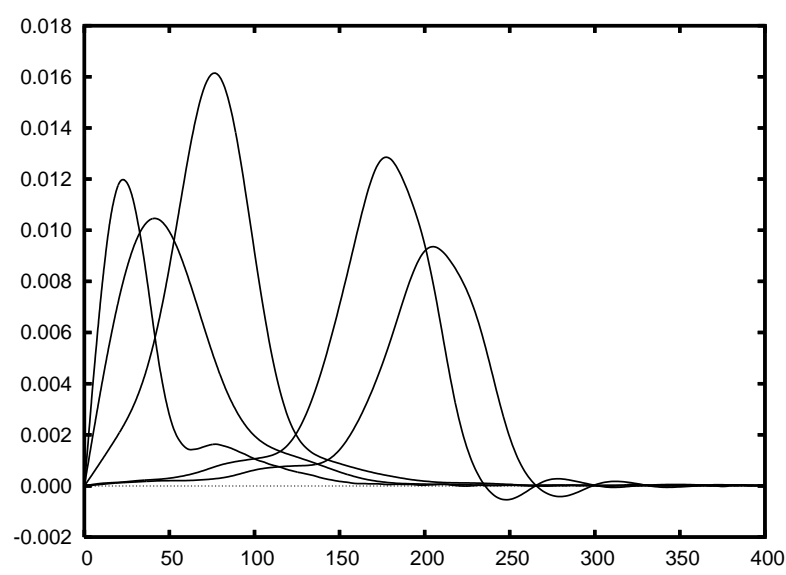

Figure 4: Irreversible parts of hysteresis loops, $\varrho_{\text {irr }}(H)$, shown in Fig. 1] The increasing annealing temperature activates the hysterons with higher coercivity. Note the regions with negative amplitudes followed by "regular" ones. Amplitudes expressed in the same, arbitrary units, as in Fig. 3 , 